\title{
Glycan profiling of gestational choriocarcinoma using a lectin microarray
}

\author{
YUSUKE KOBAYASHI $^{1}$, KENTA MASUDA ${ }^{1-3}$, KOUJI BANNO ${ }^{1}$, NANA KOBAYASHI ${ }^{2,3}$, KIYOKO UMENE $^{1}$, \\ YUYA NOGAMI $^{1}$, KOSUKE TSUJI $^{1}$, ARISA UEKI ${ }^{1-3}$, HIROYUKI NOMURA $^{1}$, KENJI SATO ${ }^{1}$, \\ EIICHIRO TOMINAGA ${ }^{1}$, TAKATSUNE SHIMIZU ${ }^{2,3}$, HIDEYUKI SAYA $^{2,3}$ and DAISUKE AOKI ${ }^{1}$ \\ ${ }^{1}$ Department of Obstetrics and Gynecology, School of Medicine, Keio University, Tokyo; \\ ${ }^{2}$ Division of Gene Regulation, Institute for Advanced Medical Research, School of Medicine, Keio University, Tokyo; \\ ${ }^{3}$ Core Research for Evolutional Science and Technology (CREST), Japan Science and Technology Agency, Tokyo, Japan
}

Received July 23, 2013; Accepted September 9, 2013

DOI: $10.3892 /$ or.2014.2979

\begin{abstract}
Glycosylation is an important post-translational modification, in which attachment of glycans to proteins has effects on biological functions and carcinogenesis. Analysis of human chorionic gonadotropin, a glycoprotein hormone produced by placental trophoblasts and trophoblastic tumors, has contributed to the diagnosis and treatment of trophoblastic disease, resulting in reduced incidence and mortality. However, alterations of the glycan structure itself in choriocarcinoma have not been characterized. We established a new choriocarcinoma cell line, induced choriocarcinoma cell-1 ( $\left.\mathrm{iC}^{3}-1\right)$, which mimics the clinical pathohistology in vivo, to examine the tumorigenesis and pathogenesis of choriocarcinoma. In this study, the alterations of glycan structures in the development of choriocarcinoma were examined by performance of comprehensive glycan profiling in clinical samples and in $\mathrm{iC}^{3}-1$ cells using a conventional microarray and the recently introduced lectin microarray. Microarray comparison showed significant upregulation of several characteristic glycogenes in the $\mathrm{iC}^{3}-1$ cells as compared to the parental HTR8/SVneo cells. The lectin array showed increased $\alpha-2-6$-sialic acid, Gal $\beta 1$ 4GlcNAc, GlcNAc $\beta 1-3$ GalNAc, and decreased $\alpha-1-6$ core fucose, high mannose, GalNac $\beta 1-4 \mathrm{Gal}$, GALNAc (Tn antigen) and Gal $\beta 1-3 \mathrm{Gal}$ in choriocarcinoma tissue compared to normal villi. This is the first report of a lectin array analysis in choriocarcinoma and provides useful information for understanding of the disease.
\end{abstract}

\section{Introduction}

Gestational choriocarcinoma, one type of gestational trophoblastic neoplasia, is a malignant epithelial trophoblastic tumor

Correspondence to: Dr Yusuke Kobayashi, Department of Obstetrics and Gynecology, School of Medicine, Keio University, 35 Shinanomachi, Shinjuku-ku, Tokyo 160-8582, Japan

E-mail:kobax@a2.keio.jp

Key words: choriocarcinoma, $\mathrm{iC}^{3}-1$, lectin microarray that can develop in the uterus after pregnancy (1). Half of the cases are preceded by a hydatidiform mole, while others occur after spontaneous abortion or normal term pregnancy (1). Treatment of a hydatidiform mole using a clinical marker, human chorionic gonadotropin (hCG), has significantly reduced the incidence of choriocarcinoma and improved survival (2). hCG is a glycoprotein that is produced by fused and differentiated placental syncytiotrophoblast cells and serves as a marker for placental function and assessment of treatment for malignant trophoblastic disease (3). The function and structure of hCG are well-established and hCG variants are known to be heavily glycosylated and sialylated glycoproteins (3).

Glycosylation is a common and versatile co- and posttranslational modification that involves attachment of glycans to proteins, lipids or other organic molecules by glycosyltransferases encoded by glycogenes $(4,5)$. Altered glycan structure by glycosylation is a common feature in cancer cells and promotes tumor cell invasion and metastasis. Certain glycans are markers for tumor progression $(6,7)$, and thus it is important to analyze the structure and binding mechanisms of these structures in the context of cancer diagnosis and treatment. However, comprehensive glycan analysis has not been performed in choriocarcinoma although several molecular studies have revealed the expression of tumor-related proteins in choriocarcinoma (1). Indeed, conventional glycan profiling tools, such as capillary electrophoresis, liquid chromatography and mass spectrometry, may not be suitable for the initial detection of glycan alterations between samples because of the time-consuming, low-throughput and requirements for complex equipment and the generation of complex data $(8,9)$. Conversely, lectin microarray has emerged in recent years and could be used for rapid and high-throughput analysis for protein glycosylation $(8,9)$.

We previously established a new choriocarcinoma cell line, induced choriocarcinoma cell-1 $\left(\mathrm{iC}^{3}-1\right)$, that mimics the clinical pathohistology in vivo, and we used these cells to examine the tumorigenesis and pathogenesis of choriocarcinoma $(10,11)$. The goal of the present study was to investigate alterations in glycan structure in the development of choriocarcinoma using comprehensive glycan profiling in clinical samples and in $\mathrm{iC}^{3}-1$ cells using a conventional microarray and the recently 
developed lectin microarray. Herein, we report the initial data from a lectin microarray in choriocarcinoma tissue.

\section{Materials and methods}

Patients and samples. Formalin-fixed, paraffin-embedded tissues from 4 cases were used in the study. First trimester villi were obtained from induced-abortion cases $(\mathrm{n}=2)$ and choriocarcinoma samples were obtained from pregnancies that had gone to term and in which hysterectomy was performed piror to other treatment $(n=2)$. The samples were obtained during surgery or delivery and were immediately fixed in formalin. All patients had been treated between 2008 and 2011 at Keio University Hospital. The samples were examined and diagnosed by two independent pathologists. This study was approved by the Institutional Review Board of Keio University School of Medicine (No. 20130115).

Cell culture. HTR8/SVneo, a human extravillous trophoblast cell line immortalized using SV40 T antigen (SV40Tag), was kindly provided by Dr C.H. Graham (Queen's University, Kingston, ON, Canada) (12). HTR8/SVneo/EGFP and $\mathrm{iC}^{3}-1$ cells were obtained as previously described (10). Before culture, cell sorting was performed with a FACSVantage SE (BD Biosciences, Franklin Lakes, NJ, USA) (10) after staining with propidium iodide (P4170; Sigma-Aldrich, St. Louis, MO, USA). Sorting based on GFP and propidium iodide fluorescence with gating on forward and side scatter was used to obtain transfected cells and exclude non-viable cells. Sorted HTR8/SVneo/EGFP and $\mathrm{iC}^{3}-1$ cells were cultured in Dulbecco's modified Eagle's medium (Gibco-BRL, Burlington, ON, Canada) supplemented with $10 \%$ heat-inactivated fetal calf serum, penicillin $(100 \mathrm{U} / \mathrm{ml})$ and streptomycin $(100 \mathrm{mg} /$ $\mathrm{ml})$ at $37^{\circ} \mathrm{C}$ in a $5 \% \mathrm{CO}_{2}$ atmosphere (10).

Microarray analysis. Microarray analysis comparing $\mathrm{iC}^{3}-1$ cells and parental HTR8/SVneo/EGFP cells were performed as previously reported (10). Briefly, total RNA from those cells was extracted using the Isogen kit (Nippon Gene, Tokyo, Japan) according to the manufacturer's instructions. After being amplified, labeled and hybridized by the Human Whole Genome Oligo Microarray kit (G4112F; Agilent, Santa Clara, CA, USA) following the Agilent user's manual protocol, the arrays were scanned using the Agilent Dual-Laser DNA Microarray Scanner (G2565A; Agilent).

Lectin microarray analysis. Lectin microarray analysis was performed as previously described (13). Briefly, the membrane fractions of HTR8/SVneo and $\mathrm{iC}^{3}-1$ cells were obtained using the ProteoExtract Subcellular Proteome Extraction kit (539790; Merck). Glycoproteins from formalin-fixed paraffinembedded tissue of normal villi and choriocarcinoma were obtained as previously reported (14). The total protein content of each sample was determined using a Micro BCA Protein Assay kit (23235; Thermo Scientific, Hemel Hempstead, UK) and adjusted to $50 \mu \mathrm{g} / \mathrm{ml}$ with phosphate-buffered saline. A portion of each sample $(1 \mu \mathrm{g})$ was added to $100 \mu \mathrm{g}$ of Cy3 monoreactive dye pack (PA23001; GE Healthcare) and incubated for $1 \mathrm{~h}$ at room temperature in the dark. Cy3-labeled proteins were desalted using a Zeba Spin Desalting Column
(89882; Thermo Scientific) and diluted to $2 \mu \mathrm{g} / \mathrm{ml}$ with probing solution (TBS containing $1 \%$ Triton X-100, $500 \mathrm{mM}$ glycine).

A sample of $100 \mu \mathrm{l}$ was added to each well on a lectin microarray glass slide (GP Biosciences, Yokohama, Japan) and incubated in a chamber ( $>80 \%$ humidity) for $150 \mathrm{~min}$ at room temperature in the dark until the binding reached equilibrium. Fluorescent images of the lectin microarrays were acquired using an evanescent-field fluorescence scanner (GlycoStation ${ }^{\mathrm{TM}}$ Reader 1200; GP Biosciences). The net intensity for each spot was calculated by subtracting the background value from the raw signal intensity, with averaging of the results from three spots. Acquired data were normalized by making the total fluorescence in each well (i.e. for 45 lectins) equivalent. All data were analyzed with Array-Pro Analyzer v. 4.5 (Media Cybernetics, Bethesda, MD, USA).

\section{Results}

Differences in glycogene expression in the ${ }^{2} C^{3}-1$ and HTR8/ SVneo cells. Gene expression profiles of $\mathrm{iC}^{3}-1$ cells and a parental trophoblast cell line, HTR8/SVneo, derived from first trimester placental tissue, were determined by microarray analysis to examine alterations in glycogenes in choriocarcinoma tumorigenesis. As previously reported, in $\mathrm{iC}^{3}-1$ cells compared to HTR8/SVneo/EGFP cells, 3,094 genes were upregulated and 3,126 genes were downregulated among the 44,000 genes examined (10). To search the differences in glycol-gene expression, we referred to the GlycoGene DataBase (http://riodb.ibase.aist.go.jp/rcmg/ggdb/) for the upregulated or downregulated genes. This analysis showed that 20 glycogenes were significantly upregulated and 15 were significantly downregulated in $\mathrm{iC}^{3}-1$ cells as compared to the HTR8/SVneo/EGFP cells (Table I). The upregulated genes included hyaluronan synthase (HAS2), which we previously found to show immunoreactivity in choriocarcinoma samples, but not in normal first trimester villi and term placenta (11).

Glycan profiling analysis in choriocarcinoma. Glycan profiling analysis of normal villi, choriocarcinoma, HTR8/SVneo and $\mathrm{iC}^{3}-1$ cells was used to investigate changes in glycan structure in the development of choriocarcinoma, using a lectin microarray. Signals for Cy3-labeled glycoproteins on the LecChip, which includes 45 different lectins, were scanned using GlycoStation Reader 1200 independently four times (Fig. 1A). The net intensity of each lectin signal was quantified using Glycostation Tools Pro and Array-Pro Analyzer (Fig. 1B). Lectins with increased levels in choriocarcinoma samples $(>20 \%$ increase in signal intensity compared to villi) were Sambucus nigra agglutinin (SNA), Sambucus sieboldiana agglutinin (SSA), Trichosanthes japonica agglutinin I (TJA-I), Ricinus communis agglutinin I (RCA120), erythroagglutinating isolectin of phytohemagglutinin (PHAE), Datura stramonium agglutinin (DSA), Agrocybe cylindracea galectin (ACG), TxLC-I, Urtica dioica agglutinin (UDA), Jacalin, and wheat germ agglutinin (WGA) (Fig. 1B and Table II). In contrast, those with decreased levels in choriocarcinoma samples ( $>20 \%$ decrease in signal intensity compared to villi) were Pisum sativum agglutinin (PSA), Lens culinaris agglutinin (LCA), Aspergillus oryzae lectin (AOL), Narcissus pseudonarcissus agglutinin (NPA), Galanthus nivalis agglutinin (GNA), Bauhinia purpurea lectin (BPL), 
Table I. Glycogenes with altered expression in $\mathrm{iC}^{3}-1$ cells as compared to HTR8/SVneo/EGFP cells.

\begin{tabular}{|c|c|c|c|}
\hline Gene symbol & Family & Fold-change & P-value \\
\hline \multicolumn{4}{|l|}{ Upregulated } \\
\hline HAS2 & Glucuronyltransferases N-acetylglucosaminyltransferases & 664.90 & 1.22.E-04 \\
\hline HS6ST2 & Sulfotransferases & 14.56 & 4.94.E-03 \\
\hline MGAT4A & $\mathrm{N}$-acetylglucosaminyltransferases & 9.18 & 1.01.E-03 \\
\hline XYLT1 & Xylosyltransferases & 9.18 & 1.05.E-03 \\
\hline GALNT14 & UDP-GalNAc:polypeptide N-acetylgalactosaminyltransferase & 8.15 & 1.13.E-03 \\
\hline POFUT1 & Fucosyltransferases & 6.62 & 3.61.E-03 \\
\hline ST6GALNAC3 & Sialyltransferases & 5.92 & 1.58.E-03 \\
\hline UST & Sulfotransferases & 4.27 & 2.38.E-03 \\
\hline GALNT3 & UDP-GalNAc:polypeptide N-acetylgalactosaminyltransferase & 3.87 & 2.89.E-03 \\
\hline ST3GAL4 & Sialyltransferases & 3.40 & 2.64.E-03 \\
\hline GCNT2 & N-acetylglucosaminyltransferases & 3.33 & 2.42.E-03 \\
\hline ST8SIA5 & Sialyltransferases & 3.11 & 3.04.E-03 \\
\hline CHST2 & Sulfotransferases & 2.70 & 5.01.E-03 \\
\hline GALNT6 & UDP-GalNAc:polypeptide N-acetylgalactosaminyltransferase & 2.65 & 5.22.E-03 \\
\hline SLC35D1 & Nucleotide sugar transporters & 2.48 & $6.01 . \mathrm{E}-03$ \\
\hline HS3ST3A1 & Sulfotransferases & 2.47 & 6.02.E-03 \\
\hline MGAT5B & $\mathrm{N}$-acetylglucosaminyltransferases & 2.26 & 1.20.E-02 \\
\hline GALNT10 & UDP-GalNAc:polypeptide N-acetylgalactosaminyltransferase & 2.20 & 7.98.E-03 \\
\hline FUT8 & Fucosyltransferases & 2.12 & 8.67.E-03 \\
\hline HAS3 & Glucuronyltransferases N-acetylglucosaminyltransferases & 2.03 & 5.27.E-03 \\
\hline \multicolumn{4}{|l|}{ Downregulated } \\
\hline B4GALNT3 & $\mathrm{N}$-acetylgalactosaminyltransferases & 0.18 & 2.32.E-03 \\
\hline ST6GALNAC2 & Sialyltransferases & 0.19 & 6.96.E-03 \\
\hline B3GALT5 & Galactosyltransferases & 0.24 & 7.09.E-03 \\
\hline GAL3ST1 & Sulfotransferases & 0.27 & 4.03.E-03 \\
\hline GALNT9 & UDP-GalNAc:polypeptide N-acetylgalactosaminyltransferase & 0.27 & 1.06.E-02 \\
\hline B3GAT1 & Glucuronyltransferases & 0.28 & 3.79.E-02 \\
\hline LARGE & Glycosyltransferase-like & 0.30 & 9.68.E-03 \\
\hline MFNG & $\mathrm{N}$-acetylglucosaminyltransferases & 0.31 & 3.78.E-03 \\
\hline CHST1 & Sulfotransferases & 0.36 & 1.58.E-03 \\
\hline ST3GAL5 & Sialyltransferases & 0.36 & 1.69.E-03 \\
\hline NDST1 & Sulfotransferases & 0.38 & 5.41.E-03 \\
\hline POMT2 & Mannosyltransferases & 0.43 & 6.87.E-03 \\
\hline CHST3 & Sulfotransferases & 0.44 & 7.25.E-03 \\
\hline B3GALT2 & Galactosyltransferases & 0.49 & 2.45.E-03 \\
\hline GALNT2 & UDP-GalNAc:polypeptide N-acetylgalactosaminyltransferase & 0.50 & 1.00.E-02 \\
\hline
\end{tabular}

Wisteria floribunda agglutinin (WFA), Amaranthus caudatus agglutinin (ACA), Helix pomatia agglutinin(HPA) and Maackia amurensis hemagglutinin (MAH) (Fig. 1B and Table II). A comparison of $\mathrm{iC}^{3}-1$ and parental HTR8/SVneo cells showed quite similar glycan profiles, suggesting that activation of the MAPK/PI3K pathway does not contribute to the alteration of glycan structures (Fig. 1C).

Based on the glycan-binding specificity of these lectins, among the N-type glycans, choriocarcinoma had higher levels of $\alpha-2-6$ sialic acids (SNA, SSA, TJA-I and WGA), reduced levels of $\alpha-1-6$ core fucoses (PSA, LCA and AOL), increases in Galß1-4GlcNAc structures (RCA120, PHAE and DSA), and decreases in high mannose (NPA and GNA), compared to normal villi (Fig. 2 and Table II). In regards to O-type glycans, choriocarcinoma had increased GlcNAc $\beta 1-3$ GalNAc structures (Jacalin) and decreases in GalNac $\beta 1-4 G a l$ (WFA), GALNAc (Tn antigen) (HPA) and Gal $\beta 1-3 \mathrm{Gal}$ (ACA), when compared to normal villi (Fig. 2 and Table II).

\section{Discussion}

The function of most proteins depends on post-translational modifications such as glycosylation, phosphorylation, methylation and sulfation (15). In glycosylation, glycan attachment modifies the function of proteins in areas such as cell recognition, cell-cell interactions and immune response, and is also 


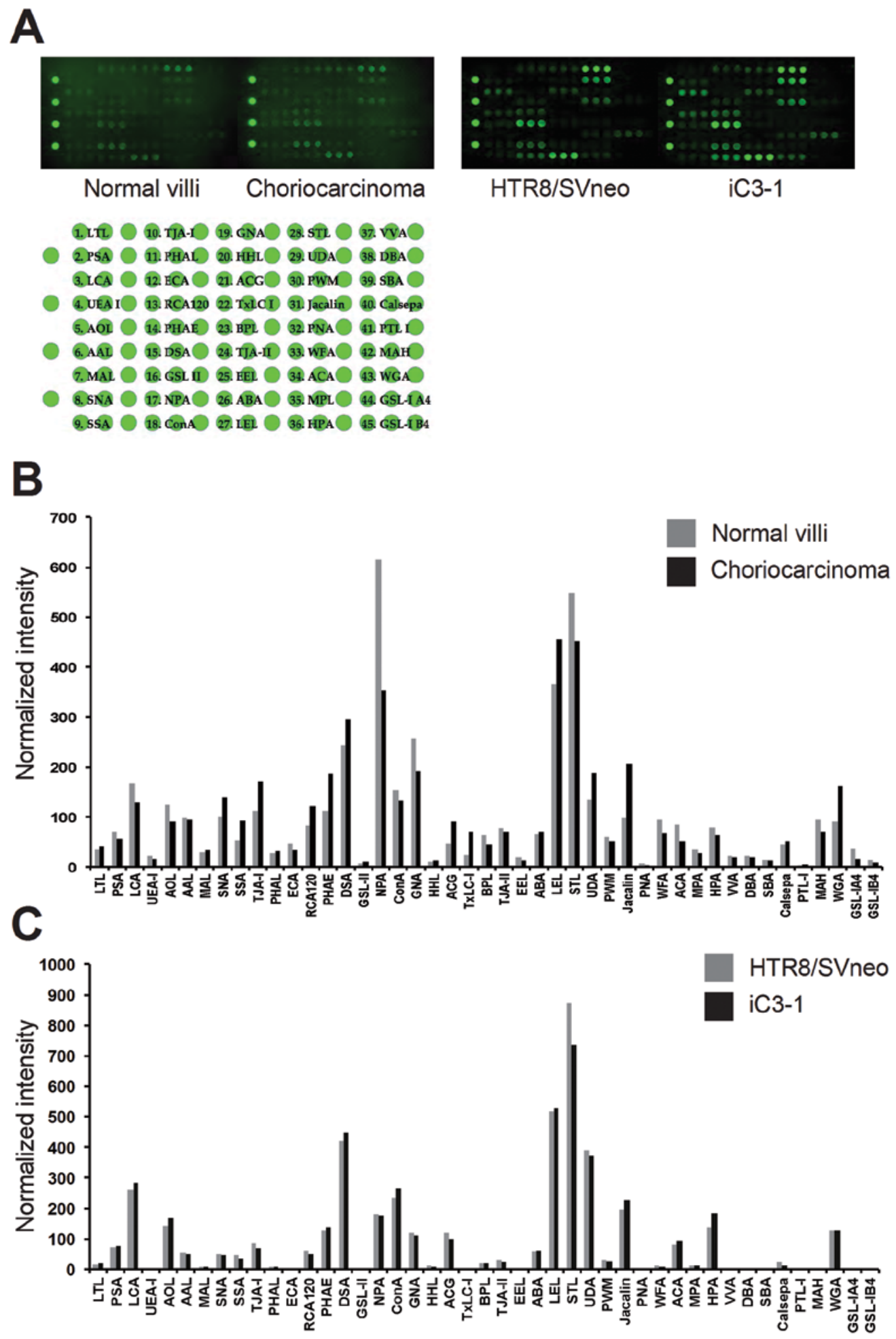

Figure 1. Comparison of glycan profiles by lectin microarray analysis of choriocarcinoma and villi samples. (A) Scans of the samples for all 45 lectins. Lectin spotting patterns are shown in the lower parts of the images. (B) Normalized glycan intensity in the samples of normal villi and choriocarcinoma. Signals were normalized using the mean of all lectins on the array. (C) Normalized glycan intensity in the samples of HTR8/SVneo and choriocarcinoma cell-1 (iC $\left.{ }^{3}-1\right)$. Signals were normalized using the mean of all lectins on the array.

important in carcinogenesis, including proliferation, invasion, metastasis and tumor progression $(16,17)$. Therefore, glycan analysis is a key to the understanding of physiological function and pathogenesis. However, progress in glycobiology has been slow due to the complexity of glycans and a lack of systematic methods with which to analyze their structures. This difficulty has been addressed by the introduction of technology for rapid, simple and high-throughput evaluation (18). In particular, the lectin microarray, based on an evanescent-field fluorescence detection principle, permits sensitive and quantitative realtime observation of multiple lectin-carbohydrate interactions unlike other conventional methods, e.g., mass spectrometry and chromatography (19). Even though a lectin microarray has emerged only in recent years, it has already been applied for 
Table II. Lectins and their glycan-binding specificity.

\begin{tabular}{|c|c|c|}
\hline Lectin & Signal ratio & Specificity \\
\hline TxLC-I & 3.010 & Man $\alpha 1-3($ Man $\alpha 1-6)$ Man, bi- and tri-antennary complex-type N-glycan, GalNAc \\
\hline Jacalin & 2.116 & Gal $\beta 1-3$ GalNAc, GalNAc \\
\hline $\mathrm{ACG}$ & 1.973 & Sia $\alpha 2-3$ Gal $\beta 1-4 G l c N A c$ \\
\hline WGA & 1.806 & Chitin oligomers, Sia \\
\hline SSA & 1.729 & Sia $\alpha 2-6 \mathrm{Gal} / \mathrm{GalNAc}$ \\
\hline PHAE & 1.666 & Bi-antennary complex-type $\mathrm{N}$-glycan with outer Gal and bisecting GlcNAc \\
\hline TJA-I & 1.533 & Sia $\alpha 2-6 \mathrm{Gal} / \mathrm{GalNAc}$ \\
\hline RCA120 & 1.455 & Gal $\beta 1-4 G l c N A c$ \\
\hline UDA & 1.408 & GlcNAc $\beta 1-4 G l c N A c$, mixture of Man5 to Man9 \\
\hline SNA & 1.379 & Sia $\alpha 2-6 \mathrm{Gal} / \mathrm{GalNAc}$ \\
\hline DSA & 1.206 & $(\mathrm{GlcNAc} \beta 1-4) n$, Gal $\beta 1-4 \mathrm{GlcNAc}$ \\
\hline NPA & 0.572 & High-Mannose, Mano1-6Man \\
\hline $\mathrm{ACA}$ & 0.608 & Gal $\beta 1-3$ GalNAc \\
\hline BPL & 0.717 & Gal $\beta 1-3$ GalNAc, GalNAc \\
\hline AOL & 0.724 & Fuc $\alpha 1-6$ GlcNAc (core fucose) \\
\hline MAH & 0.731 & Sia $\alpha 2-3 \mathrm{Gal} \beta 1-3(\operatorname{Sia} \alpha 2-6) \mathrm{GalNAc}$ \\
\hline GNA & 0.744 & High-Mannose, Man $\alpha 1-3$ Man \\
\hline LCA & 0.774 & Fuc $\alpha 1-6$ GlcNAc, $\alpha$-D-Glc, $\alpha-D-M a n$ \\
\hline PSA & 0.783 & Fuc $\alpha 1-6$ GlcNAc, $\alpha-D-G l c, \alpha-D-M a n$ \\
\hline HPA & 0.793 & $\alpha$-linked terminal GalNAc \\
\hline
\end{tabular}

ACG, Agrocybe cylindracea galectin; WGA, wheat germ agglutinin; SSA, Sambucus sieboldiana agglutinin; PHAE, erythroagglutinating isolectin of phytohemagglutinin; TJA-I, Trichosanthes japonica agglutinin I; RCA120, Ricinus communis agglutinin I; UDA, Urtica dioica agglutinin; SNA, Sambucus nigra agglutinin; DSA, Datura stramonium agglutinin; NPA, Narcissus pseudonarcissus agglutinin; ACA, Amaranthus caudatus agglutinin; BPL, Bauhinia purpurea lectin; AOL, Aspergillus oryzae lectin; MAH, Maackia amurensis hemagglutinin; GNA, Galanthus nivalis agglutinin; LCA, Lens culinaris agglutinin; PSA, Pisum sativum agglutinin; HPA, Helix pomatia agglutinin. Signal ratio, the average of net intensity in choriocarcinoma samples/that in Villi samples.

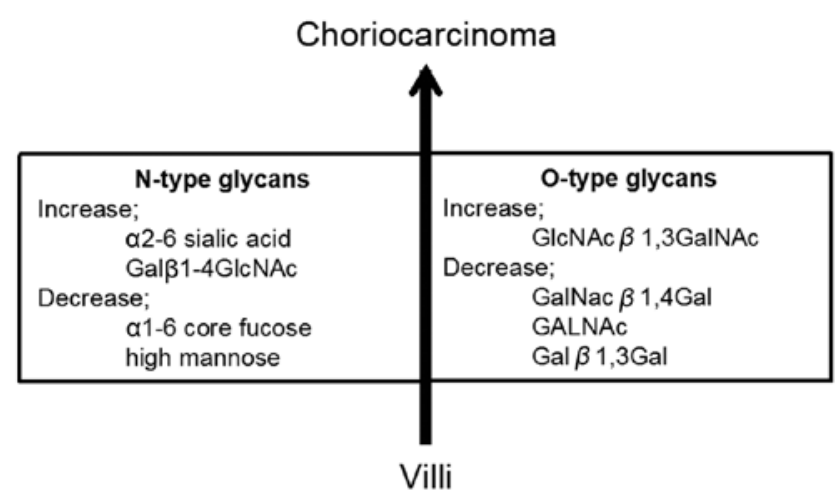

Figure 2. Alterations of glycan structure in the development of choriocarcinoma.

the development of disease-related glycoprotein markers, the evaluation of stem cells and the investigation of pluripotent cell markers (19).

Glycan analysis in choriocarcinoma has not previously been performed, in contrast to other cancers. Therefore, in the present study, we used microarrays for glycogenes and lectins in our established choriocarcinoma cell line and clinical samples to investigate glycan alterations in choriocarcinoma tumorigenesis. Conventional microarray comparison of $\mathrm{iC}^{3}-1$ and parental HTR8/SVneo cells indicated changes in several significant glycogenes. These include: HAS2, which we have shown to be significantly overexpressed in choriocarcinoma samples (10); HAS3, which is correlated with tumor growth in colon cancer and esophageal squamous cell carcinoma $(20,21)$; HS6ST2, which plays an important role in cell growth, invasion, migration and tumorigenicity in several types of cancers (22-24); FUT8, the expression of which is upregulated in lung, liver, ovarian, thyroid and colorectal cancer and correlates with tumor metastasis, disease recurrence and poor survival (25); POFUT1, which has higher expression in colon cancer and glioblastoma compared to normal tissue $(26,27)$; GalNT14, a biomarker that is predictive of the response to PARA/extrinsic pathway-targeted therapy in non-small cell lung cancer, since high levels of GalNT14 mRNA and protein in tumor cell lines are associated with Apo2L/TRAIL sensitivity (28); MGAT5B, which is upregulated in prostate cancer cells and is a marker of tumor progression (29); CHST2, a clinical molecular marker for the outcome of osteosarcoma (30); and HS3ST3A1, expression of which is higher in cancer tissue compared to normal lung tissue (31).

The lectin microarray also produced novel findings for glycans in choriocarcinoma. Among the lectins with increased or decreased levels in choriocarcinoma as compared to normal villi, SSA, TJA-I, RCA120, SNA, ACA, BPL and HPA were found to exhibit the same binding pattern in colon cancer when compared to normal tissue (14). Thus, these lectins may 
be useful as diagnostic and therapeutic markers in choriocarcinoma. In particular, the increased levels of SNA, SSA and TJA-I indicate the presence of a higher level of $\alpha-2-6$ sialic acid, and also indicate the aggressive behavior of choriocarcinoma as these lectins were found to be highly expressed in poorly differentiated endometrial cancer cells compared to well-differentiated cells (32). In addition, SSA is a potential marker for isolation of cancer stem cells (CSCs) (33). $\mathrm{iC}^{3}-1$ cells may contain components of choriocarcinoma CSCs and CD44 may be a marker for these CSCs (10). Thus, it will be of interest to attempt to isolate choriocarcinoma CSCs using CD44 and SSA.

The emerging technology of the lectin microarray is likely to permit elucidation of the precise glycan characteristics of proteins of importance in choriocarcinoma. Our data revealed that there are differences in glycogenes and lectin expression that represent potential therapeutic targets for choriocarcinoma.

\section{Acknowledgements}

We thank Charles H. Graham (Queen's University, Kingston, ON, Canada) for kindly providing the HTR8/SVneo cells; Yasunori Yoshimura (Keio University, Tokyo, Japan) for kindly donating the villi and placenta tissue; Ikuyo Ishimatsu for technical assistance in histological analyses; and Akiyoshi Noguchi for the fundamental support.

\section{References}

1. Shih IeM: Gestational trophoblastic neoplasia - pathogenesis and potential therapeutic targets. Lancet Oncol 8: 642-650, 2007.

2. Cavaliere A, Ermito S, Dinatale A and Pedata R: Management of molar pregnancy. J Prenat Med 3: 15-17, 2009.

3. Cole LA: hCG, the wonder of today's science. Reprod Biol Endocrinol 10: 24, 2012.

4. Morelle W, Canis K, Chirat F, Faid V and Michalski JC: The use of mass spectrometry for the proteomic analysis of glycosylation. Proteomics 6: 3993-4015, 2006.

5. Ohtsubo K and Marth JD: Glycosylation in cellular mechanisms of health and disease. Cell 126: 855-867, 2006.

6. Goetz JA, Mechref Y, Kang P, Jeng MH and Novotny MV: Glycomic profiling of invasive and non-invasive breast cancer cells. Glycoconj J 26: 117-131, 2009.

7. Satomaa T, Heiskanen A, Leonardsson I, et al: Analysis of the human cancer glycome identifies a novel group of tumorassociated $\mathrm{N}$-acetylglucosamine glycan antigens. Cancer Res 69: 5811-5819, 2009

8. Patwa T, Li C, Simeone DM and Lubman DM: Glycoprotein analysis using protein microarrays and mass spectrometry. Mass Spectrom Rev 29: 830-844, 2010.

9. Fry SA, Afrough B, Lomax-Browne HJ, Timms JF, Velentzis LS and Leathem AJ: Lectin microarray profiling of metastatic breast cancers. Glycobiology 21: 1060-1070, 2011.

10. Kobayashi Y, Shimizu T, Naoe H, et al: Establishment of a choriocarcinoma model from immortalized normal extravillous trophoblast cells transduced with HRASV12. Am J Pathol 179: 1471-1482, 2011.

11. Kobayashi Y, Banno K, Shimizu T, et al: Gene expression profile of a newly established choriocarcinoma cell line, $\mathrm{iC}^{3}-1$, compared to existing choriocarcinoma cell lines and normal placenta. Placenta 34: 110-118, 2013.

12. Graham CH, Hawley TS, Hawley RG, et al: Establishment and characterization of first trimester human trophoblast cells with extended lifespan. Exp Cell Res 206: 204-211, 1993.
13. Kuno A, Uchiyama N, Koseki-Kuno S, et al: Evanescent-field fluorescence-assisted lectin microarray: a new strategy for glycan profiling. Nat Methods 2: 851-856, 2005.

14. Matsuda A, Kuno A, Ishida H, Kawamoto T, Shoda J and Hirabayashi J: Development of an all-in-one technology for glycan profiling targeting formalin-embedded tissue sections. Biochem Biophys Res Commun 370: 259-263, 2008.

15. Muthana SM, Campbell CT and Gildersleeve JC: Modifications of glycans: biological significance and therapeutic opportunities. ACS Chem Biol 7: 31-43, 2012.

16. Sacchettini JC, Baum LG and Brewer CF: Multivalent proteincarbohydrate interactions. A new paradigm for supermolecular assembly and signal transduction. Biochemistry 40: 3009-3015, 2001.

17. Stanley P: Biological consequences of overexpressing or eliminating $\mathrm{N}$-acetylglucosaminyltransferase-TIII in the mouse. Biochim Biophys Acta 1573: 363-368, 2002.

18. Zhao J, Patwa TH, Lubman DM and Simeone DM: Protein biomarkers in cancer: natural glycoprotein microarray approaches. Curr Opin Mol Ther 10: 602-610, 2008.

19. Hirabayashi J, Yamada M, Kuno A and Tateno H: Lectin microarrays: concept, principle and applications. Chem Soc Rev 42: 4443-4458, 2013.

20. Teng BP, Heffler MD, Lai EC, et al: Inhibition of hyaluronan synthase-3 decreases subcutaneous colon cancer growth by increasing apoptosis. Anticancer Agents Med Chem 11: 620-628, 2011.

21. Twarock S, Freudenberger T, Poscher E, et al: Inhibition of oesophageal squamous cell carcinoma progression by in vivo targeting of hyaluronan synthesis. Mol Cancer 10: 30, 2011.

22. Song K, Li Q, Peng YB, et al: Silencing of hHS6ST2 inhibits progression of pancreatic cancer through inhibition of Notch signalling. Biochem J 436: 271-282, 2011.

23. Waaijer CJ, de Andrea CE, Hamilton A, van Oosterwijk JG, Stringer SE and Bovée JV: Cartilage tumour progression is characterized by an increased expression of heparan sulphate $6 O$-sulphation-modifying enzymes. Virchows Arch 461: 475-481, 2012.

24. Ferreras C, Rushton G, Cole CL, et al: Endothelial heparan sulfate $6-O$-sulfation levels regulate angiogenic responses of endothelial cells to fibroblast growth factor 2 and vascular endothelial growth factor. J Biol Chem 287: 36132-36146, 2012.

25. Chen CY, Jan YH, Juan YH, et al: Fucosyltransferase 8 as a functional regulator of nonsmall cell lung cancer. Proc Natl Acad Sci USA 110: 630-635, 2013.

26. Kroes RA, Dawson G and Moskal JR: Focused microarray analysis of glyco-gene expression in human glioblastomas. J Neurochem 103 (Suppl 1): S14-S24, 2007.

27. Loo LW, Tiirikainen M, Cheng I, et al: Integrated analysis of genome-wide copy number alterations and gene expression in microsatellite stable, $\mathrm{CpG}$ island methylator phenotype-negative colon cancer. Genes Chromosomes Cancer 52: 450-466, 2013.

28. Soria JC, Márk Z, Zatloukal P, et al: Randomized phase II study of dulanermin in combination with paclitaxel, carboplatin, and bevacizumab in advanced non-small-cell lung cancer. J Clin Oncol 29: 4442-4451, 2011.

29. Lange T, Ullrich S, Müller I, et al: Human prostate cancer in a clinically relevant xenograft mouse model: identification of $\beta(1,6)$-branched oligosaccharides as a marker of tumor progression. Clin Cancer Res 18: 1364-1373, 2012.

30. Chen X, Yang TT, Qiu XC, et al: Gene expression profiles of human osteosarcoma cell sublines with different pulmonary metastatic potentials. Cancer Biol Ther 11: 287-292, 2011.

31. Nakano T, Shimizu K, Kawashima O, et al: Establishment of a human lung cancer cell line with high metastatic potential to multiple organs: Gene expression associated with metastatic potential in human lung cancer. Oncol Rep 28: 1727-1735, 2012.

32. Nishijima Y, Toyoda M, Yamazaki-Inoue M, et al: Glycan profiling of endometrial cancers using lectin microarray. Genes Cells 17: 826-836, 2012.

33. Moriwaki K, Okudo K, Haraguchi N, et al: Combination use of anti-CD133 antibody and SSA lectin can effectively enrich cells with high tumorigenicity. Cancer Sci 102: 1164-1170, 2011. 